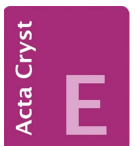

CRYSTALLOGRAPHIC COMMUNICATIONS

ISSN 2056-9890

Received 6 December 2017

Accepted 3 January 2018

Edited by M. Weil, Vienna University of Technology, Austria

Keywords: crystal structure; diphosphate; eclipsed conformation; isotypism.

CCDC reference: 1814470

Supporting information: this article has supporting information at journals.iucr.org/e

\section{Crystal structure of $\mathrm{KNaCuP}_{2} \mathrm{O}_{7}$, a new member of the diphosphate family}

\author{
Ines Fitouri and Habib Boughzala*
}

Laboratoire de Materiaux et Cristallochimie, Faculté des Sciences de Tunis, Université de Tunis El Manar, 2092 Manar II Tunis, Tunisia. *Correspondence e-mail: habib.boughzala@ipein.rnu.tn

Potassium sodium copper(II) diphosphate(V), $\mathrm{KNaCuP}_{2} \mathrm{O}_{7}$, was synthesized by solid-state reactions. It crystallizes in the $\alpha-\mathrm{Na}_{2} \mathrm{CuP}_{2} \mathrm{O}_{7}$ structure type in space group $P 2_{1} / n$. In the crystal, $\mathrm{CuO}_{5}$ square-pyramids are linked to nearly eclipsed $\mathrm{P}_{2} \mathrm{O}_{7}$ groups by sharing corners to build up corrugated layers with composition $\left[\mathrm{CuP}_{2} \mathrm{O}_{7}\right]^{2-}$ that extend parallel to $(010)$. The $\mathrm{K}^{+}$and $\mathrm{Na}^{+}$cations reside in the interlayer space and are connected to nine and seven $\mathrm{O}$ atoms, respectively. The structural model was validated by bond-valence-sum (BVS) and chargedistribution (CHARDI) analysis.

\section{Chemical context}

In order to improve the ionic conductivity in copper diphosphates with general formula $M M^{\prime} \mathrm{CuP}_{2} \mathrm{O}_{7}\left(M, M^{\prime}=\right.$ monovalent cation), we attempted to partially replace the potassium cations in $\mathrm{K}_{2} \mathrm{CuP}_{2} \mathrm{O}_{7}$ by smaller sodium cations. In the $\mathrm{K}_{2} \mathrm{CuP}_{2} \mathrm{O}_{7}$ structure, the alkali cations are located in the interlayer space between corrugated $\left[\mathrm{CuP}_{2} \mathrm{O}_{7}\right]^{2-}$ anionic layers. Reducing the size of the cation can increase its mobility, and therefore might improve the material's charge-transport behaviour.

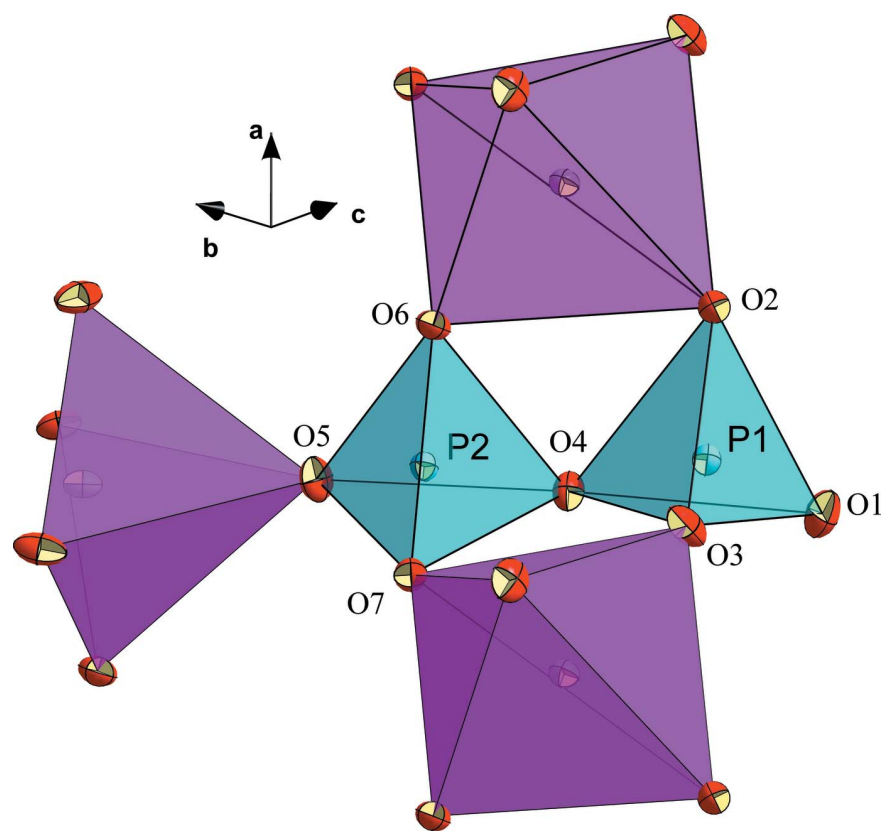

Figure 1

The diphosphate group directly connected to three $\mathrm{CuO}_{5}$ polyhedra in the structure of $\mathrm{KNaCuP}_{2} \mathrm{O}_{7}$. Displacement ellipsoids are drawn at the $50 \%$ probability level. 
Table 1

Selected bond lengths $(\AA)$.

\begin{tabular}{|c|c|c|c|}
\hline $\mathrm{Cu}-\mathrm{O} 2$ & $1.9328(18)$ & $\mathrm{Na}-\mathrm{O} 5$ & 2.398 (2) \\
\hline $\mathrm{Cu}-\mathrm{O} 3^{\mathrm{i}}$ & $1.9427(18)$ & $\mathrm{Na}-\mathrm{O}^{\mathrm{i}}$ & $2.4442(19)$ \\
\hline $\mathrm{Cu}-\mathrm{O} 6$ & $1.9743(17)$ & $\mathrm{Na}-\mathrm{O} 3^{\mathrm{iv}}$ & $2.772(2)$ \\
\hline $\mathrm{Cu}-\mathrm{O}^{\mathrm{i}}$ & $1.9872(17)$ & $\mathrm{Na}-\mathrm{O} 5^{\mathrm{i}}$ & $2.815(2)$ \\
\hline $\mathrm{Cu}-\mathrm{O}^{\mathrm{ii}}$ & $2.3225(19)$ & $\mathrm{Na}-\mathrm{O}^{\mathrm{iv}}$ & $2.878(2)$ \\
\hline $\mathrm{P} 1-\mathrm{O} 1$ & $1.482(2)$ & $\mathrm{K}-\mathrm{O} 2$ & $2.721(2)$ \\
\hline $\mathrm{P} 1-\mathrm{O} 2$ & $1.5246(19)$ & $\mathrm{K}-\mathrm{O} 5^{\mathrm{iii}}$ & $2.7245(18)$ \\
\hline $\mathrm{P} 1-\mathrm{O} 3$ & $1.5313(19)$ & $\mathrm{K}-\mathrm{O} 1^{\mathrm{i}}$ & $2.764(2)$ \\
\hline $\mathrm{P} 1-\mathrm{O} 4$ & $1.6272(17)$ & $\mathrm{K}-\mathrm{O}^{\mathrm{v}}$ & $2.7969(19)$ \\
\hline $\mathrm{P} 2-\mathrm{O} 5$ & $1.4958(17)$ & $\mathrm{K}-\mathrm{O} 7^{\mathrm{vi}}$ & 2.8450 (19) \\
\hline $\mathrm{P} 2-\mathrm{O} 6$ & $1.5252(17)$ & $\mathrm{K}-\mathrm{O} 3^{\mathrm{vii}}$ & $2.8630(18)$ \\
\hline $\mathrm{P} 2-\mathrm{O} 7$ & $1.5277(16)$ & $\mathrm{K}-\mathrm{O} 1$ & $3.036(2)$ \\
\hline $\mathrm{P} 2-\mathrm{O} 4$ & $1.6148(18)$ & $\mathrm{K}-\mathrm{O} 2^{\mathrm{vii}}$ & $3.1973(19)$ \\
\hline $\mathrm{Na}-\mathrm{O} 1^{\mathrm{iii}}$ & $2.249(2)$ & $\mathrm{K}-\mathrm{O} 3^{\mathrm{i}}$ & $3.257(2)$ \\
\hline $\mathrm{Na}-\mathrm{O} 6^{\mathrm{iv}}$ & $2.397(2)$ & & \\
\hline
\end{tabular}

Symmetry codes: (i) $x+1, y, z$; (ii) $x+\frac{1}{2},-y+\frac{3}{2}, z+\frac{1}{2}$; (iii) $-x+1,-y+1,-z$; (iv) $x+\frac{1}{2},-y+\frac{3}{2}, z-\frac{1}{2}$; (v) $\quad-x+\frac{3}{2}, y-\frac{1}{2},-z+\frac{1}{2} ; \quad$ (vi) $\quad-x+\frac{1}{2}, y-\frac{1}{2},-z+\frac{1}{2}$; $\quad$ (vii) $-x+1,-y+1,-z+1$.

Several attempts were made to prepare crystals of the hypothetical solid solution $\mathrm{K}_{2-x} \mathrm{Na}_{x} \mathrm{CuP}_{2} \mathrm{O}_{7}$, with $x$ in the range 0 to 2 . All of the attempts led to a compound with $x=1$, i.e. $\mathrm{KNaCuP}_{2} \mathrm{O}_{7}$, the crystal structure of which is reported in this communication.

\section{Structural commentary}

The title compound $\mathrm{KNaCuP}_{2} \mathrm{O}_{7}$ crystallizes isotypically with $\alpha-\mathrm{Na}_{2} \mathrm{CuP}_{2} \mathrm{O}_{7}$ (Erragh et al., 1995) and also shows resemblance with one form of $\mathrm{K}_{2} \mathrm{CuP}_{2} \mathrm{O}_{7}$ (ElMaadi et al., 1995). It is built up by corrugated $\left[\mathrm{CuP}_{2} \mathrm{O}_{7}\right]^{2-}$ layers with the alkali cations situated in the interlayer space. The anionic layers consist of a nearly eclipsed diphosphate group [O2 - P2 $-\mathrm{P} 1-$

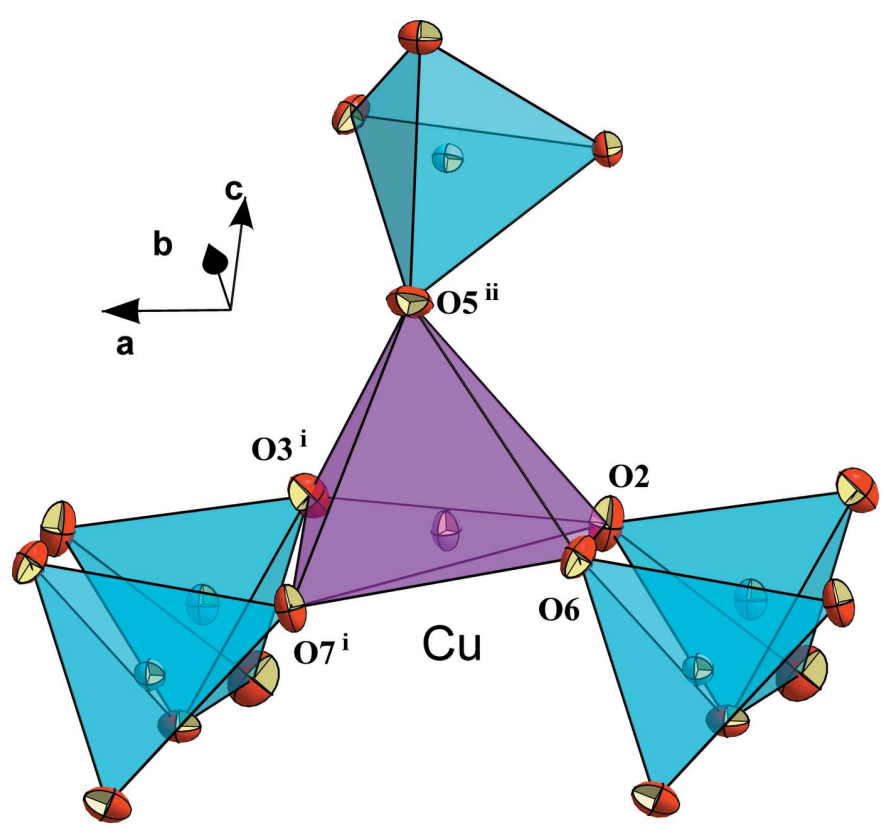

Figure 2

The $\mathrm{CuO}_{5}$ square-pyramid with neighbouring diphosphate groups in the structure of $\mathrm{KNaCuP}_{2} \mathrm{O}_{7}$. Displacement ellipsoids are drawn at the $50 \%$ probability level.
Table 2

CHARDI and BVS analysis of cation polyhedra in $\mathrm{KNaCuP}_{2} \mathrm{O}_{7}$.

\begin{tabular}{llllllll}
\hline Cation & $q i() \cdot \operatorname{sof}(i)$ & $Q(i)$ & $V(i) \cdot \operatorname{sof}(i)$ & $\mathrm{CN}(i)$ & $\operatorname{ECoN}(i)$ & $d_{\text {ar }}(i)$ & $d_{\text {med }}(i)$ \\
\hline $\mathrm{Cu}$ & 2.000 & 1.94 & 1.995 & 5 & 4.35 & 2.031 & 2.031 \\
$\mathrm{P} 1$ & 5.000 & 5.07 & 4.921 & 4 & 3.84 & 1.540 & 1.541 \\
$\mathrm{P} 2$ & 5.000 & 5.03 & 4.938 & 4 & 3.88 & 1.540 & 1.540 \\
$\mathrm{~K}$ & 1.000 & 0.98 & 1.103 & 9 & 5.20 & 2.622 & 2.564 \\
$\mathrm{Na}$ & 1.000 & 0.98 & 1.106 & 7 & 7.80 & 2.912 & 2.912 \\
\hline
\end{tabular}

Notes: $q i=$ formal oxydation number; $\operatorname{sof}(i)=$ site occupation factor; $d_{\mathrm{ar}}(i)=$ average distance; $d_{\text {med }}(i)=$ weighted average distance; $\mathrm{CN}(i)=$ coordination number; $\mathrm{ECoN}(i)=$ effective coordination number; $\sigma_{\text {cat }}=$ dispersion factor on cationic charges measuring the deviation of the computed charges; $\sigma_{\mathrm{cat}}=\left[\Sigma_{i}\left(q_{i}-Q_{i}\right)^{2} / N-1\right]^{1 / 2}=0.019$.

O6 torsion angle $\left.=15.90(1)^{\circ}\right]$ linked to $\mathrm{CuO}_{5}$ square-pyramids by sharing five of the terminal oxygen atoms $(\mathrm{O} 2, \mathrm{O} 3, \mathrm{O} 5, \mathrm{O} 6$, O7). The bridging atom $\mathrm{O} 4$ of the diphosphate unit is not involved in metal coordination, and atom $\mathrm{O} 1$ coordinates to the alkali cations in the interlayer space (Fig. 1).

The $\mathrm{P} 2-\mathrm{O} 4-\mathrm{P} 1$ bridging angle $\left[119.01(11)^{\circ}\right]$ of the diphosphate anion is close to those observed in other similar diarsenate and diphosphate structures, such as $\mathrm{KCr}_{1 / 4} \mathrm{Al}_{3 /}$ ${ }_{4} \mathrm{As}_{2} \mathrm{O}_{7}$ [118.50 (14) $)^{\circ}$ Bouhassine \& Boughzala, 2017], $\mathrm{CsCrAs}_{2} \mathrm{O}_{7}$ [118.7 (2) $)^{\circ}$ Bouhassine \& Boughzala, 2015], $\mathrm{KAlAs}_{2} \mathrm{O}_{7}$ [118.3 (2) ${ }^{\circ}$; Boughzala \& Jouini, 1995], $\alpha$ $\mathrm{Na}_{2} \mathrm{CuP}_{2} \mathrm{O}_{7}$ [118.65 (1) ${ }^{\circ}$; Erragh et al., 1995] and $\mathrm{K}_{2} \mathrm{CuP}_{2} \mathrm{O}_{7}$ [120.41 (3) ${ }^{\circ}$; ElMaadi et al., 1995]. As expected, the $\mathrm{Cu}-\mathrm{O}$ bond length to the apical oxygen atom O5 is significantly longer than the $\mathrm{Cu}-\mathrm{O}$ distances to the basal oxygen atoms of the square-pyramid (Table 1). The calculated bond-valence sum (Brown, 2002; Adams, 2003) of 1.94 valence units for the $\mathrm{Cu}$ site is in good agreement with the expected value of 2 for divalent copper. The geometry index of the $\mathrm{CuO}_{5}$ polyhedron

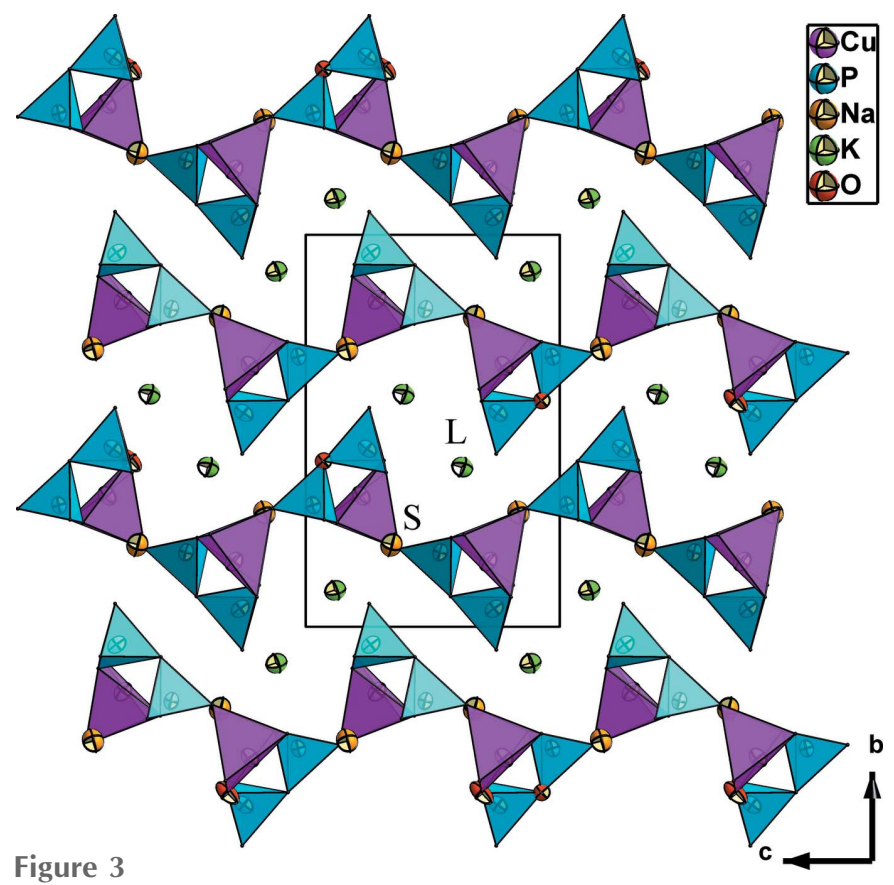

Projection of the $\mathrm{KNaCuP}_{2} \mathrm{O}_{7}$ structure along [100], showing the corrugated interlayer space housing the cations $\mathrm{K}^{+}$in the ' $L$ ' sites and $\mathrm{Na}^{+}$in the ' $S$ ' sites. Displacement ellipsoids are drawn at the $99 \%$ probability level. 
Table 3

Structural data $\left(\AA,^{\circ}\right)$ for the $M, M_{2}{ }_{2} \mathrm{CuP}_{2} \mathrm{O}_{7}$ family of compounds.

\begin{tabular}{|c|c|c|c|c|c|c|c|}
\hline Compound & Space group & $a$ & $b$ & $c$ & $\beta$ & $Z$ & Reference \\
\hline $\mathrm{Li}_{2} \mathrm{CuP}_{2} \mathrm{O}_{7}$ & $I 2 / a$ & $14.068(2)$ & $4.8600(8)$ & $8.604(1)$ & $98.97(1)$ & 4 & Spirlet et al. (1993) \\
\hline $\mathrm{Li}_{2} \mathrm{CuP}_{2} \mathrm{O}_{7}$ & $C 2 / c$ & $15.3360(14)$ & $4.8733(13)$ & $8.6259(16)$ & $114.795(10)$ & 4 & Gopalakrishna et al. (2008) \\
\hline$\alpha-\mathrm{Na}_{2} \mathrm{CuP}_{2} \mathrm{O}_{7}$ & $P 2_{1} / n$ & $8.823(3)$ & $13.494(3)$ & $5.108(2)$ & $92.77(3)$ & 4 & Erragh et al. (1995) \\
\hline$\beta-\mathrm{Na}_{2} \mathrm{CuP}_{2} \mathrm{O}_{7}$ & $C 2 / c$ & $14.715(1)$ & $5.704(2)$ & $8.066(1)$ & $115.14(1)$ & 4 & Etheredge et al. (1995) \\
\hline $\mathrm{K}_{2} \mathrm{CuP}_{2} \mathrm{O}_{7}$ & Pbnm & $9.509(4)$ & $14.389(6)$ & $5.276(2)$ & & 4 & ElMaadi et al. (1995) \\
\hline $\mathrm{K}_{2} \mathrm{CuP}_{2} \mathrm{O}_{7}$ & $P \overline{4} 2_{1} m$ & $8.056(2)$ & & $5.460(11)$ & & 2 & Keates et al. (2014) \\
\hline$\alpha-\mathrm{Rb}_{2} \mathrm{CuP}_{2} \mathrm{O}_{7}$ & Pmcn & $5.183(1)$ & $10.096(1)$ & $15.146(2)$ & & 4 & Chernyatieva et al. (2008) \\
\hline$\beta-\mathrm{Rb}_{2} \mathrm{CuP}_{2} \mathrm{O}_{7}$ & $C c$ & $7,002(1)$ & $12.751(3)$ & $9.773(2)$ & $110.93(3)$ & 4 & Shvanskaya et al. (2012) \\
\hline $\mathrm{Cs}_{2} \mathrm{CuP}_{2} \mathrm{O}_{7}$ & $C c$ & $7.460(6)$ & $12.973(10)$ & $9.980(8)$ & 111.95 & 4 & Mannasova et al. (2016) \\
\hline $\mathrm{NaCsCuP}_{2} \mathrm{O}_{7}$ & $P m n 2_{1}$ & $5.147(2)$ & $15.126(3)$ & $9.717(5)$ & & 4 & Chernyatieva et al. (2009) \\
\hline $\mathrm{Na}_{1.12} \mathrm{Ag}_{0.88} \mathrm{CuP}_{2} \mathrm{O}_{7}$ & $C 2 / c$ & $15.088(2)$ & $5.641(1)$ & $8.171(1)$ & $116.11(1)$ & 4 & Bennazha et al. (2002) \\
\hline
\end{tabular}

$\tau_{5}$, as defined by Addison et al. (1984), has a value of 0.26, indicating a distorted square-pyramidal coordination environment (the value for an ideal square-pyramid is 0 while that of an ideal trigonal bipyramid is 1). Each $\mathrm{CuO}_{5}$ polyhedron shares its vertices with two $\mathrm{P}_{2} \mathrm{O}_{7}{ }^{4-}$ anions, one of which is chelating and the other belongs to two different $\mathrm{P}_{2} \mathrm{O}_{7}$ groups (Fig. 2). This linkage leads to layers extending parallel to (010) (Fig. 3).

As in the crystal structures of $\mathrm{K}_{2} \mathrm{CuP}_{2} \mathrm{O}_{7}$ and $\alpha-\mathrm{Na}_{2} \mathrm{CuP}_{2} \mathrm{O}_{7}$, the crystal structure of $\mathrm{KNaCuP}_{2} \mathrm{O}_{7}$ exhibits two independent sites hosting the $\mathrm{K}^{+}$and $\mathrm{Na}^{+}$cations. The first site is larger (' $L$ ') and is occupied by $\mathrm{K}^{+}$cations. It is limited by two neighbouring anionic layers (Fig. 3). The resulting $\mathrm{KO}_{9}$ coordination polyhedron is considerably distorted (Fig. 4, Table 1). Its bond-valence sum is 0.98 valence units (Table 2).

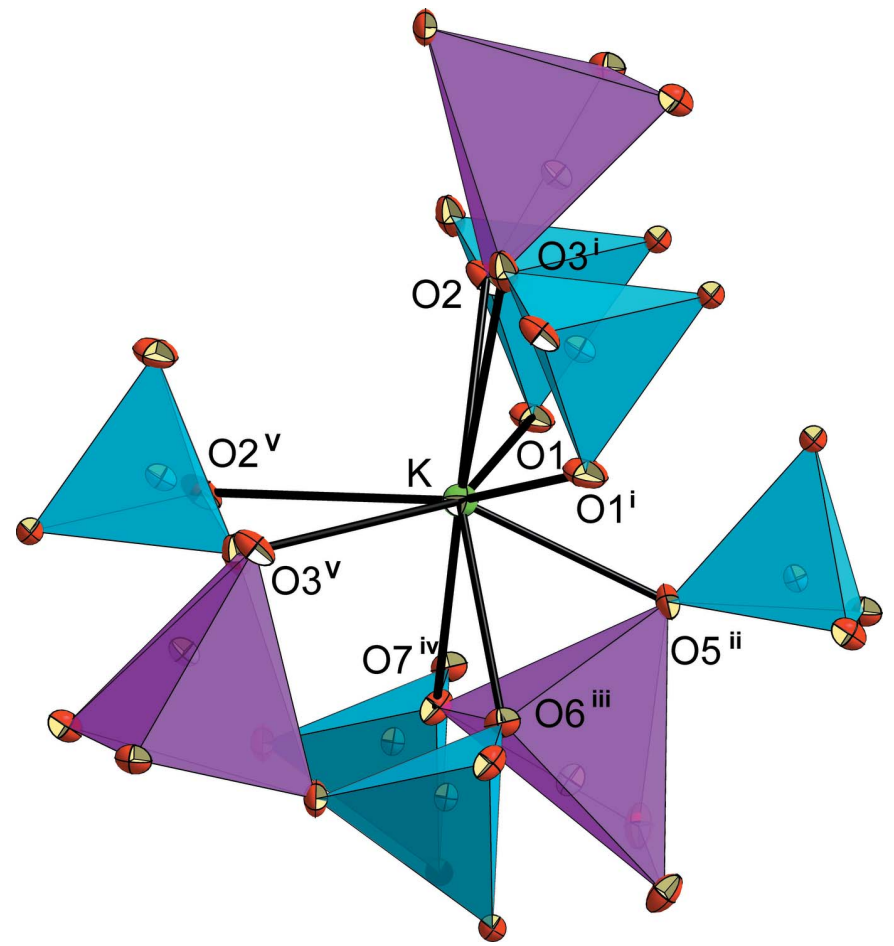

Figure 4

The nine-coordinated $\mathrm{K}^{+}$cation in the large ' $L$ ' site within the interlayer space in the structure of $\mathrm{KNaCuP}_{2} \mathrm{O}_{7}$. Displacement ellipsoids are drawn at $50 \%$ probability level. [Symmetry codes: (i) $1+x, y, z$; (ii) $1-x, 1-y$, $-z$; (iii) $\frac{3}{2}-x,-\frac{1}{2}+y, \frac{1}{2}-z$; (iv) $\frac{1}{2}-x,-\frac{1}{2}+y, \frac{1}{2}-z$; (v) $1-x, 1-y, 1-z$.]
The second site is smaller (' $S$ ') and is occupied by $\mathrm{Na}^{+}$cations. It is surrounded by three $\mathrm{CuO}_{5}$ and five $\mathrm{PO}_{4}$ polyhedra, delimiting an ellipsoidal cavity as shown in Figs. 3 and 5. The irregular coordination sphere of $\mathrm{Na}^{+}$is made up of seven oxygen atoms and shows an effective coordination number (ECoN; Nespolo et al., 2001) of 5.2 (for other ECoN values, see Table 2). The $\mathrm{Na}-\mathrm{O}$ bonds lengths can be divided in groups of four short [2.249 (2)-2.4442 (19) §] and three long [2.772 (2)-2.878 (2) $\AA$ ] bonds (Table 1). Its bond-valence sum is 0.98 valence units (Table 2 ).

\section{Database survey}

Table 3 summarizes lattice parameters and the symmetry of related $M M^{\prime} \mathrm{CuP}_{2} \mathrm{O}_{7}\left(M, M^{\prime}=\right.$ monovalent cation $)$ compounds

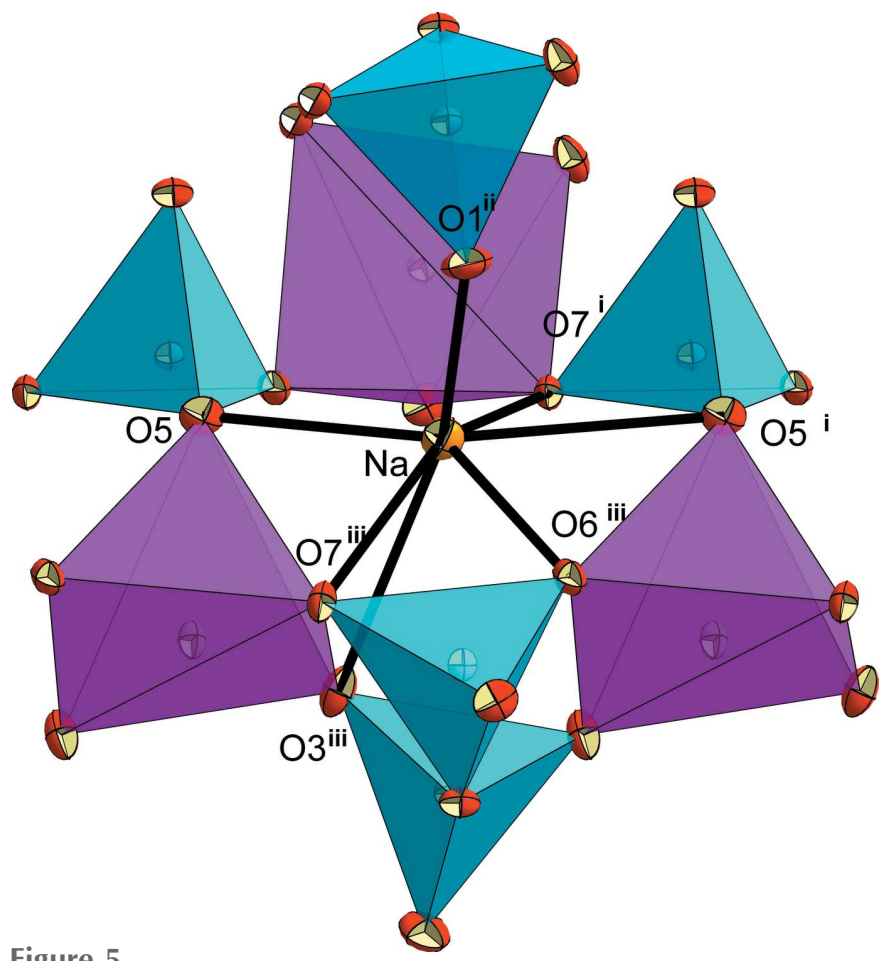

Figure 5

The surrounding of the seven-coordinated $\mathrm{Na}^{+}$cation in the ' $S$ ' site in the structure of $\mathrm{KNaCuP}_{2} \mathrm{O}_{7}$. Displacement ellipsoids are drawn at $50 \%$ probability level. [Symmetry codes: (i) $1+x, y, z$; (ii) $1-x, 1-y,-z$; (iii) $\left.\frac{1}{2}+x, \frac{3}{2}-y,-\frac{1}{2}+z\right]$. 
Table 4

Experimental details.

\begin{tabular}{|c|c|}
\hline \multicolumn{2}{|l|}{ Crystal data } \\
\hline Chemical formula & $\mathrm{KNaCuP}_{2} \mathrm{O}_{7}$ \\
\hline$M_{\mathrm{r}}$ & 299.57 \\
\hline Crystal system, space group & Monoclinic, $P 2_{1} / n$ \\
\hline Temperature (K) & 298 \\
\hline$a, b, c(\AA)$ & $5.176(3), 13.972(5), 9.067$ (3) \\
\hline$\beta\left({ }^{\circ}\right)$ & $91.34(2)$ \\
\hline$V\left(\AA^{3}\right)$ & $655.6(5)$ \\
\hline$Z$ & 4 \\
\hline Radiation type & Мо $K \alpha$ \\
\hline$\mu\left(\mathrm{mm}^{-1}\right)$ & 4.51 \\
\hline Crystal size $(\mathrm{mm})$ & $0.18 \times 0.13 \times 0.09$ \\
\hline \multicolumn{2}{|l|}{ Data collection } \\
\hline Diffractometer & Enraf-Nonius CAD-4 \\
\hline Absorption correction & $\psi$ scan (North et al., 1968) \\
\hline$T_{\min }, T_{\max }$ & $0.868,0.997$ \\
\hline $\begin{array}{l}\text { No. of measured, independent and } \\
\text { observed }[I>2 \sigma(I)] \text { reflections }\end{array}$ & $3300,1425,1291$ \\
\hline$R_{\text {int }}$ & 0.036 \\
\hline$(\sin \theta / \lambda)_{\max }\left(\AA^{-1}\right)$ & 0.638 \\
\hline \multicolumn{2}{|l|}{ Refinement } \\
\hline$R\left[F^{2}>2 \sigma\left(F^{2}\right)\right], w R\left(F^{2}\right), S$ & $0.020,0.059,1.01$ \\
\hline No. of reflections & 1425 \\
\hline No. of parameters & 110 \\
\hline$\Delta \rho_{\max }, \Delta \rho_{\min }\left(\mathrm{e} \AA^{-3}\right)$ & $0.48,-0.42$ \\
\hline
\end{tabular}

Computer programs: CAD-4 EXPRESS (Enraf-Nonius, 1994), XCAD4 (Harms \& Wocadlo, 1995), SHELXS97 (Sheldrick, 2008), SHELXL2014/7 (Sheldrick, 2015), DIAMOND (Brandenburg, 2008) and publCIF (Westrip, 2010).

compiled in the ICSD (ICSD, 2017). It is apparent that the size of the cation(s) defines the structure type.

\section{Synthesis and crystallization}

Crystals of $\mathrm{KNaCuP}_{2} \mathrm{O}_{7}$ were obtained from a mixture of $\mathrm{KH}_{2} \mathrm{PO}_{4}, \mathrm{NaH}_{2} \mathrm{PO}_{4}$ and $\mathrm{CuO}$ in the molar ratio $\mathrm{K}: \mathrm{Na}: \mathrm{Cu}: \mathrm{P}=$ 1:1:2:2. The stoichiometric mixture was finely ground and heated in a porcelain crucible at $623 \mathrm{~K}$ for $12 \mathrm{~h}$ to eliminate volatile products. The temperature was then increased to $873 \mathrm{~K}$ and held for $15 \mathrm{~d}$ until fusion was reached. The sample was slowly cooled $\left(5 \mathrm{~K} \mathrm{~d}^{-1}\right)$ to $500 \mathrm{~K}$ and finally allowed to cool radiatively to room temperature. The product was washed with water and rinsed with an aqueous solution of $\mathrm{HCl}$ (low concentration). Only one type of regular light-blue prismatic crystals was observed. The obtained crystals were ground and checked by powder X-ray diffraction. Rietveld analysis with the program TOPAS 4.2 (Coelho, 2009) revealed a single-phase product of $\mathrm{KNaCuP}_{2} \mathrm{O}_{7}$.

\section{Refinement}

Crystal data, data collection and structure refinement details are summarized in Table 4. The occupancy of the $\mathrm{Na}^{+}$and $\mathrm{K}^{+}$ sites was checked by independent refinement of the site occupation factors. In each case, full occupancy was observed without the contribution of the other cation. The maximum and minimum electron densities remaining in the difference-
Fourier map are located $0.67 \AA$ from $\mathrm{O} 7$ and $0.48 \AA$ from $\mathrm{Cu}$, respectively.

\section{Acknowledgements}

We acknowledge the assistance of the staff of the Tunisian Laboratory of Materials and Crystallography during the data collection.

\section{Funding information}

Funding for this research was provided by: Ministère de l'enseignement supérieur et de la recherche scientifique (Tunisie).

\section{References}

Adams, S. (2003). softBV. University of Göttingen, Germany.

Addison, A. W., Rao, T. N., Reedijk, J., van Rijn, J. \& Verschoor, G. C. (1984). J. Chem. Soc. Dalton Trans. pp. 1349-1356.

Bennazha, J., Boukhari, A. \& Holt, E. M. (2002). Acta Cryst. C58, i87i89.

Boughzala, H. \& Jouini, T. (1995). Acta Cryst. C51, 179-181.

Bouhassine, M. A. \& Boughzala, H. (2015). Acta Cryst. E71, 636-639.

Bouhassine, M. A. \& Boughzala, H. (2017). Acta Cryst. E73, 345-348.

Brandenburg, K. (2008). DIAMOND. Crystal Impact GbR, Bonn, Germany.

Brown, I. D. (2002). The Chemical Bond in Inorganic Chemistry: The Bond Valence Model. Oxford University Press.

Chernyatieva, A. P., Krivovichev, S. V. \& Spiridonova, D. V. (2008). Book of Abstracts, VI International Conference on Inorganic Materials, pp. 3-143. Dresden: Elsevier.

Chernyatieva, A. P., Krivovichev, S. V. \& Spiridonova, D. V. (2009). Proceedings of the XX Russian Conference of Young Scientists in Memory of the Corresponding Member of the Academy of Sciences of the USSR, K. O. Kratts.

Coelho, A. A. (2009). TOPAS 4.2. www. topas-academic. net

ElMaadi, A., Boukhari, A. \& Holt, E. M. (1995). J. Alloys Compd. 223, 13-17.

Enraf-Nonius (1994). CAD-4 EXPRESS. Enraf-Nonius, Delft, The Netherlands.

Erragh, F., Boukhari, A., Abraham, F. \& Elouadi, B. (1995). J. Solid State Chem. 120, 23-31.

Etheredge, K. M. S. \& Hwu, S. J. (1995). Inorg. Chem. 34, 1495-1499.

Gopalakrishna, G. S., Mahesh, M. J., Ashamanjari, K. G. \& Prasad, J. S. (2008). Mater. Res. Bull. 43, 1171-1178.

Harms, K. \& Wocadlo, S. (1995). XCAD4. University of Marburg, Germany.

ICSD (2017). Inorganic Crystal Structure Database. FIZ-Karlsruhe, Germany. http://www.fiz-karlsruhe. de/fiz/products/icsd/welcome. html

Keates, A. C., Wang, Q. \& Weller, M. T. (2014). J. Solid State Chem. 210, 10-14.

Mannasova, A. A., Chernyatieva, A. P. \& Krivovichev, S. V. (2016). Z. Kristallogr. 231, 65-69.

Nespolo, M., Ferraris, G., Ivaldi, G. \& Hoppe, R. (2001). Acta Cryst. B57, 652-664.

North, A. C. T., Phillips, D. C. \& Mathews, F. S. (1968). Acta Cryst. A24, 351-359.

Sheldrick, G. M. (2008). Acta Cryst. A64, 112-122.

Sheldrick, G. M. (2015). Acta Cryst. C71, 3-8.

Shvanskaya, L. V., Yakubovich, O. V. \& Urusov, V. S. (2012). Dokl. Phys. Chem. 442, 19-26.

Spirlet, M. R., Rebizant, J. \& Liegeois-Duyckaerts, M. (1993). Acta Cryst. C49, 209-211.

Westrip, S. P. (2010). J. Appl. Cryst. 43, 920-925. 


\section{supporting information}

Acta Cryst. (2018). E74, 109-112］https://doi.org/10.1107/S2056989018000130]

\section{Crystal structure of $\mathrm{KNaCuP}_{2} \mathrm{O}_{7}$, a new member of the diphosphate family}

\section{Ines Fitouri and Habib Boughzala}

\section{Computing details}

Data collection: CAD-4 EXPRESS (Enraf-Nonius, 1994); cell refinement: CAD-4 EXPRESS (Enraf-Nonius, 1994); data reduction: XCAD4 (Harms \& Wocadlo, 1995); program(s) used to solve structure: SHELXS97 (Sheldrick, 2008); program(s) used to refine structure: SHELXL2014/7 (Sheldrick, 2015); molecular graphics: DIAMOND (Brandenburg, 2008); software used to prepare material for publication: publCIF (Westrip, 2010).

Potassium sodium copper(II) diphosphate(V)

Crystal data

$\mathrm{KNaCuP}_{2} \mathrm{O}_{7}$

$M_{r}=299.57$

Monoclinic, $P 2_{1} / n$

$a=5.176(3) \AA$

$b=13.972(5) \AA$

$c=9.067(3) \AA$

$\beta=91.34(2)^{\circ}$

$V=655.6(5) \AA^{3}$

$Z=4$

Data collection

Enraf-Nonius CAD-4

diffractometer

Radiation source: fine-focus sealed tube

$\omega / 2 \theta$ scans

Absorption correction: $\psi$ scan

(North et al., 1968)

$T_{\min }=0.868, T_{\max }=0.997$

3300 measured reflections

1425 independent reflections

\section{Refinement}

Refinement on $F^{2}$

Least-squares matrix: full

$R\left[F^{2}>2 \sigma\left(F^{2}\right)\right]=0.020$

$w R\left(F^{2}\right)=0.059$

$S=1.01$

1425 reflections

110 parameters

0 restraints

Primary atom site location: structure-invariant direct methods
$F(000)=580$

$D_{\mathrm{x}}=3.035 \mathrm{Mg} \mathrm{m}^{-3}$

Mo $K \alpha$ radiation, $\lambda=0.71073 \AA$

Cell parameters from 25 reflections

$\theta=10-15^{\circ}$

$\mu=4.51 \mathrm{~mm}^{-1}$

$T=298 \mathrm{~K}$

Prism, blue

$0.18 \times 0.13 \times 0.09 \mathrm{~mm}$

1291 reflections with $I>2 \sigma(I)$

$R_{\text {int }}=0.036$

$\theta_{\max }=27.0^{\circ}, \theta_{\min }=2.7^{\circ}$

$h=-6 \rightarrow 6$

$k=-2 \rightarrow 17$

$l=-11 \rightarrow 11$

2 standard reflections every $120 \mathrm{~min}$ intensity decay: $1.1 \%$

Secondary atom site location: difference Fourier map

$w=1 /\left[\sigma^{2}\left(F_{\mathrm{o}}^{2}\right)+(0.0261 P)^{2}+0.7316 P\right]$

where $P=\left(F_{\mathrm{o}}{ }^{2}+2 F_{\mathrm{c}}{ }^{2}\right) / 3$

$(\Delta / \sigma)_{\max }=0.001$

$\Delta \rho_{\max }=0.48$ e $\AA^{-3}$

$\Delta \rho_{\min }=-0.42$ e $\AA^{-3}$

Extinction correction: SHELXL-2014/7

(Sheldrick, 2015),

$\mathrm{Fc}^{*}=\mathrm{kFc}\left[1+0.001 \mathrm{xFc}^{2} \lambda^{3} / \sin (2 \theta)\right]^{-1 / 4}$

Extinction coefficient: 0.0120 (9) 


\section{Special details}

Geometry. All esds (except the esd in the dihedral angle between two 1.s. planes) are estimated using the full covariance matrix. The cell esds are taken into account individually in the estimation of esds in distances, angles and torsion angles; correlations between esds in cell parameters are only used when they are defined by crystal symmetry. An approximate (isotropic) treatment of cell esds is used for estimating esds involving l.s. planes.

Refinement. Refinement of $\mathrm{F}^{2}$ against ALL reflections. The weighted R-factor $\mathrm{wR}$ and goodness of fit $\mathrm{S}$ are based on $\mathrm{F}^{2}$, conventional $\mathrm{R}$-factors $\mathrm{R}$ are based on $\mathrm{F}$, with $\mathrm{F}$ set to zero for negative $\mathrm{F}^{2}$. The threshold expression of $\mathrm{F}^{2}>2 \operatorname{sigma}\left(\mathrm{F}^{2}\right)$ is used only for calculating R-factors(gt) etc. and is not relevant to the choice of reflections for refinement. R-factors based on $\mathrm{F}^{2}$ are statistically about twice as large as those based on F, and R- factors based on ALL data will be even larger.

Fractional atomic coordinates and isotropic or equivalent isotropic displacement parameters $\left(\hat{A}^{2}\right)$

\begin{tabular}{lllll}
\hline & $x$ & $y$ & $z$ & $U_{\text {iso }} * / U_{\text {eq }}$ \\
\hline $\mathrm{Cu}$ & $0.76039(5)$ & $0.66590(2)$ & $0.21888(3)$ & $0.01093(12)$ \\
$\mathrm{P} 1$ & $0.26527(12)$ & $0.54589(5)$ & $0.24314(7)$ & $0.01115(15)$ \\
$\mathrm{P} 2$ & $0.25245(11)$ & $0.68972(4)$ & $0.02910(6)$ & $0.00898(15)$ \\
$\mathrm{Na}$ & $0.7521(2)$ & $0.70912(8)$ & $-0.16272(11)$ & $0.0192(2)$ \\
$\mathrm{K}$ & $0.75717(11)$ & $0.40697(4)$ & $0.38439(6)$ & $0.01850(15)$ \\
$\mathrm{O} 1$ & $0.2223(4)$ & $0.44115(15)$ & $0.2502(2)$ & $0.0223(4)$ \\
$\mathrm{O} 2$ & $0.5278(3)$ & $0.57557(14)$ & $0.3077(2)$ & $0.0176(4)$ \\
$\mathrm{O} 3$ & $0.0576(3)$ & $0.60643(15)$ & $0.31633(19)$ & $0.0179(4)$ \\
$\mathrm{O} 4$ & $0.2588(3)$ & $0.57729(12)$ & $0.07029(18)$ & $0.0126(4)$ \\
$\mathrm{O} 5$ & $0.2938(3)$ & $0.69884(14)$ & $-0.13300(18)$ & $0.0160(4)$ \\
$\mathrm{O} 6$ & $0.4685(3)$ & $0.73465(13)$ & $0.12308(19)$ & $0.0138(4)$ \\
$\mathrm{O} 7$ & $-0.0125(3)$ & $0.72712(13)$ & $0.07246(19)$ & $0.0139(4)$ \\
\hline
\end{tabular}

Atomic displacement parameters $\left(\AA^{2}\right)$

\begin{tabular}{lllllll}
\hline & $U^{11}$ & $U^{22}$ & $U^{33}$ & $U^{12}$ & $U^{13}$ & $U^{23}$ \\
\hline $\mathrm{Cu}$ & $0.00808(16)$ & $0.01226(18)$ & $0.01244(17)$ & $0.00043(11)$ & $0.00010(11)$ & $0.00296(11)$ \\
$\mathrm{P} 1$ & $0.0103(3)$ & $0.0102(3)$ & $0.0130(3)$ & $0.0000(2)$ & $0.0007(2)$ & $0.0029(2)$ \\
$\mathrm{P} 2$ & $0.0088(3)$ & $0.0099(3)$ & $0.0082(3)$ & $0.0002(2)$ & $0.0008(2)$ & $0.0009(2)$ \\
$\mathrm{Na}$ & $0.0190(5)$ & $0.0213(6)$ & $0.0172(5)$ & $-0.0021(4)$ & $-0.0001(4)$ & $0.0020(5)$ \\
$\mathrm{K}$ & $0.0220(3)$ & $0.0153(3)$ & $0.0182(3)$ & $0.0022(2)$ & $-0.0008(2)$ & $0.0000(2)$ \\
$\mathrm{O} 1$ & $0.0272(10)$ & $0.0115(10)$ & $0.0280(10)$ & $-0.0046(8)$ & $0.0001(8)$ & $0.0049(8)$ \\
$\mathrm{O} 2$ & $0.0123(8)$ & $0.0198(10)$ & $0.0206(9)$ & $-0.0026(8)$ & $-0.0022(7)$ & $0.0093(8)$ \\
$\mathrm{O} 3$ & $0.0151(9)$ & $0.0261(10)$ & $0.0126(8)$ & $0.0075(8)$ & $0.0008(7)$ & $0.0036(8)$ \\
$\mathrm{O} 4$ & $0.0178(8)$ & $0.0093(8)$ & $0.0107(8)$ & $-0.0006(7)$ & $0.0012(6)$ & $-0.0003(7)$ \\
$\mathrm{O} 5$ & $0.0213(9)$ & $0.0179(9)$ & $0.0088(8)$ & $0.0015(8)$ & $0.0025(7)$ & $0.0013(7)$ \\
$\mathrm{O} 6$ & $0.0115(8)$ & $0.0115(8)$ & $0.0181(8)$ & $-0.0002(7)$ & $-0.0041(6)$ & $0.0011(7)$ \\
$\mathrm{O} 7$ & $0.0091(8)$ & $0.0154(9)$ & $0.0173(8)$ & $0.0016(7)$ & $0.0030(6)$ & $0.0044(8)$ \\
\hline
\end{tabular}

Geometric parameters $\left(A,{ }^{\circ}\right)$

\begin{tabular}{llll}
\hline $\mathrm{Cu}-\mathrm{O} 2$ & $1.9328(18)$ & $\mathrm{Na}-\mathrm{P} 2^{\mathrm{i}}$ & $3.0977(12)$ \\
$\mathrm{Cu}-\mathrm{O} 3^{\mathrm{i}}$ & $1.9427(18)$ & $\mathrm{Na}-\mathrm{P} 2^{\text {viii }}$ & $3.1316(12)$ \\
$\mathrm{Cu}-\mathrm{O} 6$ & $1.9743(17)$ & $\mathrm{Na}-\mathrm{Cu}^{\mathrm{ix}}$ & $3.2481(11)$ \\
$\mathrm{Cu}-\mathrm{O} 7^{\mathrm{i}}$ & $1.9872(17)$ & $\mathrm{Na}-\mathrm{Cu}^{\text {viii }}$ & $3.3550(11)$ \\
$\mathrm{Cu}-\mathrm{O} 5^{\mathrm{ii}}$ & $2.3225(19)$ & $\mathrm{K}-\mathrm{O} 2$ & $2.721(2)$
\end{tabular}




$$
\begin{aligned}
& \mathrm{Cu}-\mathrm{Na}^{\mathrm{ii}} \\
& \mathrm{Cu}-\mathrm{Na}^{\text {iii }} \\
& \mathrm{Cu}-\mathrm{K}^{\mathrm{iv}} \\
& \mathrm{Cu}-\mathrm{Na} \\
& \mathrm{Cu}-\mathrm{K} \\
& \mathrm{P} 1-\mathrm{O} 1 \\
& \mathrm{P} 1-\mathrm{O} 2 \\
& \mathrm{P} 1-\mathrm{O} 3 \\
& \mathrm{P} 1-\mathrm{O} 4 \\
& \mathrm{P} 1-\mathrm{K} \\
& \mathrm{P} 1-\mathrm{K}^{\mathrm{v}} \\
& \mathrm{P} 1-\mathrm{K}^{\mathrm{vi}} \\
& \mathrm{P} 2-\mathrm{O} 5 \\
& \mathrm{P} 2-\mathrm{O} 6 \\
& \mathrm{P} 2-\mathrm{O} 7 \\
& \mathrm{P} 2-\mathrm{O} 4 \\
& \mathrm{P} 2-\mathrm{Na}^{\mathrm{vi}} \\
& \mathrm{P} 2-\mathrm{Na}^{\mathrm{iii}} \\
& \mathrm{P} 2-\mathrm{Na} \\
& \mathrm{Na}-\mathrm{O}^{\text {vii }} \\
& \mathrm{Na}-\mathrm{O}^{\text {viii }} \\
& \mathrm{Na}-\mathrm{O} 5 \\
& \mathrm{Na}-\mathrm{O}^{\mathrm{i}} \\
& \mathrm{Na}-\mathrm{O}^{\text {viii }} \\
& \mathrm{Na}-\mathrm{O} 5^{\mathrm{i}} \\
& \mathrm{Na}-\mathrm{O} 7^{\text {viii }} \\
& \mathrm{O} 2-\mathrm{Cu}-\mathrm{O}^{\mathrm{i}} \\
& \mathrm{O} 2-\mathrm{Cu}-\mathrm{O} 6 \\
& \mathrm{O} 3{ }^{\mathrm{i}}-\mathrm{Cu}-\mathrm{O} 6 \\
& \mathrm{O} 2-\mathrm{Cu}-\mathrm{O}^{\mathrm{i}} \\
& \mathrm{O}^{\mathrm{i}}-\mathrm{Cu}-\mathrm{O}^{\mathrm{i}} \\
& \mathrm{O} 6-\mathrm{Cu}-\mathrm{O}^{\mathrm{i}} \\
& \mathrm{O} 2-\mathrm{Cu}-\mathrm{O} 5^{\mathrm{ii}} \\
& \mathrm{O} 3^{\mathrm{i}}-\mathrm{Cu}-\mathrm{O}^{\mathrm{ii}} \\
& \mathrm{O} 6-\mathrm{Cu}-\mathrm{O} 5^{\mathrm{ii}} \\
& \mathrm{O} 7^{\mathrm{i}}-\mathrm{Cu}-\mathrm{O}^{\mathrm{ii}} \\
& \mathrm{O} 2-\mathrm{Cu}-\mathrm{Na}^{\mathrm{ii}} \\
& \mathrm{O} 3-\mathrm{Cu}-\mathrm{Na}^{\mathrm{ii}} \\
& \mathrm{O} 6-\mathrm{Cu}-\mathrm{Na}^{\mathrm{ii}} \\
& \mathrm{O} 7-\mathrm{Cu}-\mathrm{Na}^{\mathrm{ii}} \\
& \mathrm{O} 5^{\mathrm{ii}}-\mathrm{Cu}-\mathrm{Na}^{\mathrm{ii}} \\
& \mathrm{O} 2-\mathrm{Cu}-\mathrm{Na}^{\mathrm{iii}} \\
& \mathrm{O} 3^{\mathrm{i}}-\mathrm{Cu}-\mathrm{Na}^{\mathrm{iii}} \\
& \mathrm{O} 6-\mathrm{Cu}-\mathrm{Na}^{\mathrm{iii}} \\
& \mathrm{O} 7^{\mathrm{i}}-\mathrm{Cu}-\mathrm{Na}^{\mathrm{iii}} \\
& \mathrm{O} 5^{\mathrm{ii}}-\mathrm{Cu}-\mathrm{Na}^{\mathrm{iii}} \\
& \mathrm{Na}^{\mathrm{ii}}-\mathrm{Cu}-\mathrm{Na}^{\mathrm{iii}}
\end{aligned}
$$

$3.2481(11)$

$3.3550(11)$

$3.4966(7)$

$3.5117(10)$

3.9169 (7)

$1.482(2)$

$1.5246(19)$

$1.5313(19)$

$1.6272(17)$

$3.4260(9)$

$3.4455(9)$

$3.5329(9)$

$1.4958(17)$

$1.5252(17)$

$1.5277(16)$

$1.6148(18)$

$3.0977(12)$

$3.1316(12)$

3.1617 (12)

$2.249(2)$

$2.397(2)$

$2.398(2)$

2.4442 (19)

$2.772(2)$

$2.815(2)$

$2.878(2)$

$91.46(8)$

$91.34(7)$

$176.22(8)$

$160.62(8)$

$90.77(7)$

$87.43(7)$

$109.22(7)$

$92.15(8)$

$84.52(7)$

$89.93(7)$

134.76 (6)

58.24 (7)

118.00 (6)

$61.20(5)$

47.48 (5)

72.89 (6)

134.08 (6)

$44.76(5)$

118.06 (6)

$55.94(5)$

$103.22(3)$

$$
\begin{aligned}
& \mathrm{K}-\mathrm{O}^{\mathrm{vii}} \\
& \mathrm{K}-\mathrm{O}^{\mathrm{i}} \\
& \mathrm{K}-\mathrm{O}^{\mathrm{x}} \\
& \mathrm{K}-\mathrm{O}^{\mathrm{xi}} \\
& \mathrm{K}-3^{\mathrm{v}} \\
& \mathrm{K}-\mathrm{O} 1 \\
& \mathrm{~K}-\mathrm{O} 2^{\mathrm{v}} \\
& \mathrm{K}-\mathrm{O} 3^{\mathrm{i}} \\
& \mathrm{K}-\mathrm{P} 1^{\mathrm{v}} \\
& \mathrm{K}-\mathrm{Cu}^{\mathrm{x}} \\
& \mathrm{O} 1-\mathrm{Na}^{\mathrm{vii}} \\
& \mathrm{O} 1-\mathrm{K}^{\mathrm{vi}} \\
& \mathrm{O} 2-\mathrm{K}^{\mathrm{v}} \\
& \mathrm{O} 3-\mathrm{Cu}^{\mathrm{vi}} \\
& \mathrm{O} 3-\mathrm{Na}^{\mathrm{iii}} \\
& \mathrm{O} 3-\mathrm{K}^{\mathrm{v}} \\
& \mathrm{O} 3-\mathrm{K}^{\mathrm{vi}} \\
& \mathrm{O} 5-\mathrm{Cu}^{\mathrm{ix}} \\
& \mathrm{O} 5-\mathrm{K}^{\mathrm{vii}} \\
& \mathrm{O} 5-\mathrm{Na}^{\mathrm{vi}} \\
& \mathrm{O} 6-\mathrm{Na}^{\mathrm{iii}} \\
& \mathrm{O} 6-\mathrm{K}^{\mathrm{iv}} \\
& \mathrm{O} 7-\mathrm{Cu}^{\mathrm{vi}} \\
& \mathrm{O} 7-\mathrm{Na}^{\mathrm{vi}} \\
& \mathrm{O} 7-\mathrm{K}^{\mathrm{xii}} \\
& \mathrm{O} 7-\mathrm{Na}^{\mathrm{iii}}
\end{aligned}
$$

2.7245 (18)

2.764 (2)

2.7969 (19)

2.8450 (19)

2.8630 (18)

3.036 (2)

3.1973 (19)

3.257 (2)

3.4455 (9)

3.4965 (7)

2.249 (2)

2.764 (2)

3.1973 (19)

1.9427 (18)

2.772 (2)

2.8630 (18)

3.257 (2)

2.3225 (19)

2.7245 (18)

2.815 (2)

2.397 (2)

2.7968 (19)

1.9872 (17)

2.4441 (19)

2.8451 (19)

2.878 (2)

$45.56(5)$

127.14 (6)

36.58 (4)

146.13 (6)

37.24 (4)

150.57 (4)

58.62 (2)

65.37 (2)

108.65 (6)

35.44 (4)

148.47 (6)

81.19 (5)

$77.32(5)$

43.13 (4)

86.18 (4)

$64.76(2)$

57.50 (2)

151.44 (4)

$103.22(3)$

102.87 (6)

$96.73(6)$ 


$$
\begin{aligned}
& \mathrm{O} 2-\mathrm{Cu}-\mathrm{K}^{\mathrm{iv}} \\
& \mathrm{O} 3-\mathrm{Cu}-\mathrm{K}^{\mathrm{iv}} \\
& \mathrm{O} 6-\mathrm{Cu}-\mathrm{K}^{\mathrm{iv}} \\
& \mathrm{O} 7^{\mathrm{i}}-\mathrm{Cu}-\mathrm{K}^{\mathrm{iv}} \\
& \mathrm{O} 5^{\mathrm{ii}}-\mathrm{Cu}-\mathrm{K}^{\mathrm{iv}} \\
& \mathrm{Na}{ }^{\mathrm{ii}}-\mathrm{Cu}-\mathrm{K}^{\mathrm{iv}} \\
& \mathrm{Na}^{\mathrm{iii}}-\mathrm{Cu}-\mathrm{K}^{\mathrm{iv}} \\
& \mathrm{O} 2-\mathrm{Cu}-\mathrm{Na} \\
& \mathrm{O} 3-\mathrm{Cu}-\mathrm{Na} \\
& \mathrm{O} 6-\mathrm{Cu}-\mathrm{Na} \\
& \mathrm{O} 7-\mathrm{Cu}-\mathrm{Na} \\
& \mathrm{O} 5^{\mathrm{ii}}-\mathrm{Cu}-\mathrm{Na} \\
& \mathrm{Na}{ }^{\mathrm{ii}}-\mathrm{Cu}-\mathrm{Na} \\
& \mathrm{Na}^{\mathrm{iii}}-\mathrm{Cu}-\mathrm{Na} \\
& \mathrm{K}^{\mathrm{iv}}-\mathrm{Cu}-\mathrm{Na} \\
& \mathrm{O} 2-\mathrm{Cu}-\mathrm{K} \\
& \mathrm{O} 3^{\mathrm{i}}-\mathrm{Cu}-\mathrm{K} \\
& \mathrm{O} 6-\mathrm{Cu}-\mathrm{K} \\
& \mathrm{O} 7-\mathrm{Cu}-\mathrm{K} \\
& \mathrm{O} 55^{\mathrm{ii}}-\mathrm{Cu}-\mathrm{K} \\
& \mathrm{Na}^{\mathrm{ii}}-\mathrm{Cu}-\mathrm{K} \\
& \mathrm{Na}^{\mathrm{iii}}-\mathrm{Cu}-\mathrm{K} \\
& \mathrm{K}-\mathrm{iv}-\mathrm{Cu}-\mathrm{K} \\
& \mathrm{Na}-\mathrm{Cu}-\mathrm{K} \\
& \mathrm{O} 1-\mathrm{P} 1-\mathrm{O} 2 \\
& \mathrm{O} 1-\mathrm{P} 1-\mathrm{O} 3 \\
& \mathrm{O} 2-\mathrm{P} 1-\mathrm{O} 3 \\
& \mathrm{O} 1-\mathrm{P} 1-\mathrm{O} 4 \\
& \mathrm{O} 2-\mathrm{P} 1-\mathrm{O} 4 \\
& \mathrm{O} 3-\mathrm{P} 1-\mathrm{O} 4 \\
& \mathrm{O} 1-\mathrm{P} 1-\mathrm{K} \\
& \text { O2- } 1 \text { 1-K } \\
& \text { O3- } 1-\mathrm{K} \\
& \text { O4-P1-K } \\
& \mathrm{O} 1-\mathrm{P} 1-\mathrm{K}^{\mathrm{v}} \\
& \mathrm{O} 2-\mathrm{P} 1-\mathrm{K}^{\mathrm{v}} \\
& \mathrm{O} 3-\mathrm{P} 1-\mathrm{K}^{\mathrm{v}} \\
& \text { O4- } \mathrm{P} 1-\mathrm{K}^{\mathrm{v}} \\
& \mathrm{K}-\mathrm{P} 1-\mathrm{K}^{\mathrm{v}} \\
& \mathrm{O} 1-\mathrm{P} 1-\mathrm{K}^{\mathrm{vi}} \\
& \mathrm{O} 2-\mathrm{P} 1-\mathrm{K}^{\mathrm{vi}} \\
& \mathrm{O} 3-\mathrm{P} 1-\mathrm{K}^{\mathrm{vi}} \\
& \mathrm{O} 4-\mathrm{P} 1-\mathrm{K}^{\mathrm{vi}} \\
& \mathrm{K}-\mathrm{P} 1-\mathrm{K}^{\mathrm{vi}} \\
& \mathrm{K}^{\mathrm{v}}-\mathrm{P} 1-\mathrm{K}^{\mathrm{vi}} \\
& \mathrm{O} 5-\mathrm{P} 2-\mathrm{O} 6 \\
& \mathrm{O} 5-\mathrm{P} 2-\mathrm{O} 7 \\
& \mathrm{O} 6-\mathrm{P} 2-\mathrm{O} 7
\end{aligned}
$$

$136.72(6)$

\begin{tabular}{|c|c|}
\hline $\mathrm{O}^{\mathrm{vii}}-\mathrm{K}-\mathrm{O} 1^{\mathrm{i}}$ & $78.10(6)$ \\
\hline $\mathrm{O} 2-\mathrm{K}-\mathrm{O} 6^{\mathrm{x}}$ & $163.30(6)$ \\
\hline $\mathrm{O}^{\mathrm{vii}}-\mathrm{K}-\mathrm{O}^{\mathrm{x}}$ & $63.38(5)$ \\
\hline $\mathrm{O} 1^{\mathrm{i}}-\mathrm{K}-\mathrm{O}^{\mathrm{x}}$ & $71.95(6)$ \\
\hline $\mathrm{O} 2-\mathrm{K}-\mathrm{O} 7^{\mathrm{xi}}$ & $127.36(6)$ \\
\hline $\mathrm{O}^{\mathrm{vii}}-\mathrm{K}-\mathrm{O}^{\mathrm{xi}}$ & $66.49(5)$ \\
\hline $\mathrm{O} 1^{\mathrm{i}}-\mathrm{K}-\mathrm{O} 7^{\mathrm{xi}}$ & $127.46(6)$ \\
\hline $\mathrm{O} 6^{\mathrm{x}}-\mathrm{K}-\mathrm{O} 7^{\mathrm{xi}}$ & $58.05(5)$ \\
\hline $\mathrm{O} 2-\mathrm{K}-\mathrm{O}^{\mathrm{v}}$ & $115.68(6)$ \\
\hline $\mathrm{O}^{\mathrm{vii}}-\mathrm{K}-\mathrm{O}^{\mathrm{v}}$ & $141.38(6)$ \\
\hline $\mathrm{O} 1^{\mathrm{i}}-\mathrm{K}-\mathrm{O} 3^{\mathrm{v}}$ & $98.78(6)$ \\
\hline $\mathrm{O} 6^{\mathrm{x}}-\mathrm{K}-\mathrm{O} 3^{\mathrm{v}}$ & $78.91(6)$ \\
\hline $\mathrm{O} 7^{\mathrm{xi}}-\mathrm{K}-\mathrm{O}^{\mathrm{v}}$ & $87.26(6)$ \\
\hline $\mathrm{O} 2-\mathrm{K}-\mathrm{O} 1$ & $51.17(5)$ \\
\hline $\mathrm{O} 5^{\mathrm{vii}-\mathrm{K}}-\mathrm{O} 1$ & $71.36(6)$ \\
\hline $\mathrm{O} 11^{\mathrm{i}-\mathrm{K}}-\mathrm{O} 1$ & $126.29(7)$ \\
\hline $\mathrm{O} 6^{\mathrm{x}}-\mathrm{K}-\mathrm{O} 1$ & $125.76(6)$ \\
\hline $\mathrm{O} 7^{\mathrm{xi}}-\mathrm{K}-\mathrm{O} 1$ & $77.84(5)$ \\
\hline $\mathrm{O}^{\mathrm{v}}-\mathrm{K}-\mathrm{O} 1$ & $132.24(6)$ \\
\hline $\mathrm{O} 2-\mathrm{K}-\mathrm{O} 2^{\mathrm{v}}$ & $87.12(5)$ \\
\hline $\mathrm{O} 5^{\mathrm{vii}}-\mathrm{K}-\mathrm{O} 2^{\mathrm{v}}$ & $137.25(6)$ \\
\hline $\mathrm{O} 1^{\mathrm{i}}-\mathrm{K}-\mathrm{O} 2^{\mathrm{v}}$ & $142.70(6)$ \\
\hline $\mathrm{O} 6^{\mathrm{x}}-\mathrm{K}-\mathrm{O} 2^{\mathrm{v}}$ & $109.37(5)$ \\
\hline $\mathrm{O} 7^{\mathrm{xi}}-\mathrm{K}-\mathrm{O} 2^{\mathrm{v}}$ & $74.42(5)$ \\
\hline $\mathrm{O} 3^{\mathrm{v}}-\mathrm{K}-\mathrm{O} 2^{\mathrm{v}}$ & $47.81(5)$ \\
\hline $\mathrm{O} 1-\mathrm{K}-\mathrm{O} 2^{\mathrm{v}}$ & $84.43(5)$ \\
\hline $\mathrm{O} 2-\mathrm{K}-\mathrm{O} 3^{\mathrm{i}}$ & $54.42(5)$ \\
\hline $\mathrm{O} 5^{\mathrm{vii}-\mathrm{K}}-\mathrm{O} 3^{\mathrm{i}}$ & $110.06(5)$ \\
\hline $\mathrm{O} 1^{\mathrm{i}}-\mathrm{K}-\mathrm{O} 3^{\mathrm{i}}$ & $49.03(5)$ \\
\hline $\mathrm{O} 6^{\mathrm{x}}-\mathrm{K}-\mathrm{O} 3^{\mathrm{i}}$ & $119.15(5)$ \\
\hline $\mathrm{O} 7^{\mathrm{xi}}-\mathrm{K}-\mathrm{O} 3^{\mathrm{i}}$ & $176.12(5)$ \\
\hline $\mathrm{O} 3^{\mathrm{v}}-\mathrm{K}-\mathrm{O} 3^{\mathrm{i}}$ & $94.86(5)$ \\
\hline $\mathrm{O} 1-\mathrm{K}-\mathrm{O} 3^{\mathrm{i}}$ & $102.97(5)$ \\
\hline $\mathrm{O} 2^{\mathrm{v}}-\mathrm{K}-\mathrm{O} 3^{\mathrm{i}}$ & $109.40(5)$ \\
\hline $\mathrm{O} 2-\mathrm{K}-\mathrm{P} 1$ & $25.58(4)$ \\
\hline $\mathrm{O} 5^{\mathrm{vii}}-\mathrm{K}-\mathrm{P} 1$ & $86.44(4)$ \\
\hline $\mathrm{O} 1 \mathrm{i}-\mathrm{K}-\mathrm{P} 1$ & $112.63(5)$ \\
\hline $\mathrm{O}^{\mathrm{x}}-\mathrm{K}-\mathrm{P} 1$ & $148.42(4)$ \\
\hline $\mathrm{O} 7^{\mathrm{xi}}-\mathrm{K}-\mathrm{P} 1$ & $102.86(4)$ \\
\hline $\mathrm{O} 3^{\mathrm{v}}-\mathrm{K}-\mathrm{P} 1$ & $128.38(4)$ \\
\hline $\mathrm{O} 1-\mathrm{K}-\mathrm{P} 1$ & $25.61(4)$ \\
\hline $\mathrm{O} 2^{\mathrm{v}}-\mathrm{K}-\mathrm{P} 1$ & $85.94(4)$ \\
\hline $\mathrm{O} 3^{\mathrm{i}}-\mathrm{K}-\mathrm{P} 1$ & $78.37(3)$ \\
\hline $\mathrm{O} 2-\mathrm{K}-\mathrm{P} 1^{\mathrm{v}}$ & $93.47(4)$ \\
\hline $\mathrm{O} 5^{\mathrm{vii}}-\mathrm{K}-\mathrm{P} 1^{\mathrm{v}}$ & $156.78(4)$ \\
\hline $\mathrm{O} 1^{\mathrm{i}}-\mathrm{K}-\mathrm{P} 1^{\mathrm{v}}$ & $116.62(5)$ \\
\hline $\mathrm{O} 6^{\mathrm{x}}-\mathrm{K}-\mathrm{P} 1^{\mathrm{v}}$ & $102.54(4)$ \\
\hline $\mathrm{O} 7^{\mathrm{xi}}-\mathrm{K}-\mathrm{P} 1^{\mathrm{v}}$ & $90.50(4)$ \\
\hline
\end{tabular}

$123.31(6)$

$53.04(5)$

$54.45(5)$

$51.11(5)$

$65.54(2)$

$64.45(2)$

$121.97(6)$

$120.87(5)$

$59.42(5)$

$42.40(5)$

$115.40(5)$

$102.98(2)$

$103.54(2)$

$64.59(2)$

$39.55(6)$

$56.02(6)$

$127.43(5)$

$131.41(5)$

$122.07(5)$

$112.35(2)$

110.35 (2)

172.759 (11)

$122.43(2)$

$112.67(12)$

$114.78(12)$

$108.17(11)$

$107.95(11)$

$107.13(10)$

$105.64(10)$

$62.31(8)$

$50.40(8)$

$132.38(7)$

$120.75(7)$

$97.92(9)$

$67.78(7)$

$55.22(7)$

153.15 (7)

77.51 (2)

47.78 (8)

132.43 (7)

$67.06(8)$

119.91 (7)

96.10 (2)

$72.974(19)$

$113.20(10)$

$111.96(10)$

$111.49(10)$ 


$$
\begin{aligned}
& \mathrm{O} 5-\mathrm{P} 2-\mathrm{O} 4 \\
& \mathrm{O} 6-\mathrm{P} 2-\mathrm{O} 4 \\
& \mathrm{O} 7-\mathrm{P} 2-\mathrm{O} 4 \\
& \mathrm{O} 5-\mathrm{P} 2-\mathrm{Na}^{\mathrm{vi}} \\
& \mathrm{O} 6-\mathrm{P} 2-\mathrm{Na}^{\mathrm{vi}} \\
& \mathrm{O} 7-\mathrm{P} 2-\mathrm{Na}^{\mathrm{vi}} \\
& \mathrm{O} 4-\mathrm{P} 2-\mathrm{Na}^{\mathrm{vi}} \\
& \mathrm{O} 5-\mathrm{P} 2-\mathrm{Na}^{\mathrm{iii}} \\
& \mathrm{O} 6-\mathrm{P} 2-\mathrm{Na}^{\mathrm{iii}} \\
& \mathrm{O} 7-\mathrm{P} 2-\mathrm{Na}^{\mathrm{iii}} \\
& \mathrm{O} 4-\mathrm{P} 2-\mathrm{Na}^{\mathrm{iii}} \\
& \mathrm{Na}^{\mathrm{vi}}-\mathrm{P} 2-\mathrm{Na}^{\mathrm{iii}} \\
& \mathrm{O} 5-\mathrm{P} 2-\mathrm{Na} \\
& \mathrm{O} 6-\mathrm{P} 2-\mathrm{Na} \\
& \mathrm{O} 7-\mathrm{P} 2-\mathrm{Na} \\
& \mathrm{O} 4-\mathrm{P} 2-\mathrm{Na} \\
& \mathrm{Na}^{\mathrm{vi}}-\mathrm{P} 2-\mathrm{Na} \\
& \mathrm{Na}^{\mathrm{iii}}-\mathrm{P} 2-\mathrm{Na} \\
& \mathrm{O} 1^{\text {vii }-\mathrm{Na}-\mathrm{O} 6^{\text {viii }}} \\
& \mathrm{O} 1{ }^{\mathrm{vii}}-\mathrm{Na}-\mathrm{O} 5 \\
& \mathrm{O}^{\text {viii- }-\mathrm{Na}-\mathrm{O} 5} \\
& \mathrm{O} 1^{\mathrm{vii}}-\mathrm{Na}-\mathrm{O} 7^{\mathrm{i}}
\end{aligned}
$$

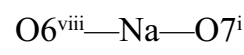

$$
\begin{aligned}
& \mathrm{O} 5-\mathrm{Na}-\mathrm{O}^{\mathrm{i}} \\
& \mathrm{O} 1^{\text {vii }-\mathrm{Na}-\mathrm{O} 3^{\text {viii }}} \\
& \mathrm{O}^{\text {viii- }}-\mathrm{Na}-\mathrm{O}^{\text {viii }} \\
& \mathrm{O} 5-\mathrm{Na}-\mathrm{O}^{\text {viii }} \\
& \mathrm{O}^{\mathrm{i}}-\mathrm{Na}-\mathrm{O}^{\text {viii }}
\end{aligned}
$$

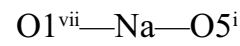

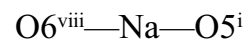

$$
\begin{aligned}
& \mathrm{O} 5-\mathrm{Na}-\mathrm{O}^{\mathrm{i}} \\
& \mathrm{O} 7-\mathrm{Na}-\mathrm{O}^{\mathrm{i}} \\
& \mathrm{O} 3^{\text {viii- }-\mathrm{Na}-\mathrm{O} 5^{\mathrm{i}}}
\end{aligned}
$$

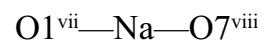

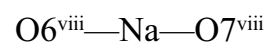

$$
\begin{aligned}
& \mathrm{O} 5-\mathrm{Na}-\mathrm{O} 7^{\text {viii }} \\
& \mathrm{O} 7^{\mathrm{i}}-\mathrm{Na}-\mathrm{O} 7^{\text {viii }}
\end{aligned}
$$

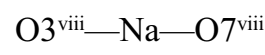

$$
\begin{aligned}
& \mathrm{O} 5^{\mathrm{i}}-\mathrm{Na}-\mathrm{O} 7^{\text {viii }}
\end{aligned}
$$

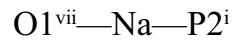

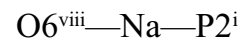

$$
\begin{aligned}
& \mathrm{O} 5-\mathrm{Na}-\mathrm{P} 2^{\mathrm{i}} \\
& \mathrm{O} 7^{\mathrm{i}}-\mathrm{Na}-\mathrm{P}^{\mathrm{i}}
\end{aligned}
$$

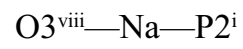

$$
\begin{aligned}
& \mathrm{O} 5^{\mathrm{i}}-\mathrm{Na}-\mathrm{P} 2^{\mathrm{i}}
\end{aligned}
$$

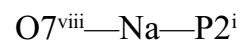

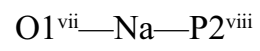

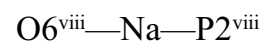

$107.91(10)$

$105.10(10)$

$106.66(10)$

$65.03(7)$

$150.48(8)$

$51.00(7)$

$103.13(7)$

$147.30(9)$

$48.05(7)$

$66.21(7)$

$103.48(7)$

$116.33(3)$

$46.71(7)$

$70.95(7)$

$149.66(7)$

$101.43(7)$

$111.56(3)$

$118.02(3)$

$89.29(8)$

\begin{tabular}{|c|c|}
\hline $\mathrm{O} 3^{\mathrm{v}}-\mathrm{K}-\mathrm{P} 1^{\mathrm{v}}$ & $26.06(4)$ \\
\hline $\mathrm{O} 1-\mathrm{K}-\mathrm{P} 1^{\mathrm{v}}$ & $108.15(4)$ \\
\hline $\mathrm{O} 2^{\mathrm{v}}-\mathrm{K}-\mathrm{P} 1^{\mathrm{v}}$ & $26.19(3)$ \\
\hline $\mathrm{O} 3^{\mathrm{i}}-\mathrm{K}-\mathrm{P} 1^{\mathrm{v}}$ & $92.83(4)$ \\
\hline $\mathrm{P} 1-\mathrm{K}-\mathrm{P} 1^{\mathrm{v}}$ & $102.49(2)$ \\
\hline $\mathrm{O} 2-\mathrm{K}-\mathrm{Cu}^{\mathrm{x}}$ & $139.21(4)$ \\
\hline $\mathrm{O}^{\mathrm{vii}}-\mathrm{K}-\mathrm{Cu}^{\mathrm{x}}$ & $41.57(4)$ \\
\hline $\mathrm{O} 1^{\mathrm{i}}-\mathrm{K}-\mathrm{Cu}^{\mathrm{x}}$ & $93.78(5)$ \\
\hline $\mathrm{O} 6^{\mathrm{x}}-\mathrm{K}-\mathrm{Cu}^{\mathrm{x}}$ & $34.34(3)$ \\
\hline $\mathrm{O}^{7 \mathrm{xi}}-\mathrm{K}-\mathrm{Cu}^{\mathrm{x}}$ & $34.63(3)$ \\
\hline $\mathrm{O} 3^{\mathrm{v}}-\mathrm{K}-\mathrm{Cu}^{\mathrm{x}}$ & $101.36(5)$ \\
\hline $\mathrm{O} 1-\mathrm{K}-\mathrm{Cu}^{\mathrm{x}}$ & $91.51(4)$ \\
\hline $\mathrm{O} 2^{\mathrm{v}}-\mathrm{K}-\mathrm{Cu}^{\mathrm{x}}$ & $107.32(4)$ \\
\hline $\mathrm{O}^{\mathrm{i}}-\mathrm{K}-\mathrm{Cu}^{\mathrm{x}}$ & 141.53 \\
\hline $\mathrm{P} 1-\mathrm{K}-\mathrm{Cu}^{\mathrm{x}}$ & $115.54(2)$ \\
\hline $\mathrm{P} 1^{\mathrm{v}}-\mathrm{K}-\mathrm{Cu}^{\mathrm{x}}$ & $116.45(2)$ \\
\hline $\mathrm{P} 1-\mathrm{O} 1-\mathrm{Na}^{\mathrm{vii}}$ & $153.68(14)$ \\
\hline $\mathrm{P} 1-\mathrm{O} 1-\mathrm{K}^{\mathrm{vi}}$ & $108.83(11)$ \\
\hline $\mathrm{Na}^{\mathrm{vii}}-\mathrm{O} 1-\mathrm{K}^{\mathrm{vi}}$ & $93.05(7)$ \\
\hline $\mathrm{P} 1-\mathrm{O} 1-\mathrm{K}$ & $92.08(9)$ \\
\hline $\mathrm{Na}^{\mathrm{vii}}-\mathrm{O} 1-\mathrm{K}$ & $86.20(7)$ \\
\hline $\mathrm{K}^{\mathrm{vi}-\mathrm{O} 1-\mathrm{K}}$ & $126.29(7)$ \\
\hline $\mathrm{P} 1-\mathrm{O} 2-\mathrm{Cu}$ & $125.16(11)$ \\
\hline $\mathrm{P} 1-\mathrm{O} 2-\mathrm{K}$ & $104.02(10)$ \\
\hline $\mathrm{Cu}-\mathrm{O} 2-\mathrm{K}$ & $113.55(8)$ \\
\hline $\mathrm{P} 1-\mathrm{O} 2-\mathrm{K}^{\mathrm{v}}$ & $86.03(8)$ \\
\hline $\mathrm{Cu}-\mathrm{O} 2-\mathrm{K}^{\mathrm{v}}$ & $128.21(9)$ \\
\hline $\mathrm{K}-\mathrm{O} 2-\mathrm{K}^{\mathrm{v}}$ & $92.88(5)$ \\
\hline $\mathrm{P} 1-\mathrm{O} 3-\mathrm{Cu}^{\mathrm{vi}}$ & $126.56(11)$ \\
\hline $\mathrm{P} 1-\mathrm{O} 3-\mathrm{Na}^{\mathrm{iii}}$ & $106.61(10)$ \\
\hline $\mathrm{Cu}^{\mathrm{vi}}-\mathrm{O} 3-\mathrm{Na}^{\mathrm{iii}}$ & $85.17(8)$ \\
\hline $\mathrm{P} 1-\mathrm{O} 3-\mathrm{K}^{\mathrm{v}}$ & $98.72(8)$ \\
\hline $\mathrm{Cu}^{\mathrm{vi}}-\mathrm{O} 3-\mathrm{K}^{\mathrm{v}}$ & $134.68(8)$ \\
\hline $\mathrm{Na}^{\mathrm{iii}}-\mathrm{O} 3-\mathrm{K}^{\mathrm{v}}$ & $83.27(6)$ \\
\hline $\mathrm{P} 1-\mathrm{O} 3-\mathrm{K}^{\mathrm{vi}}$ & $87.28(9)$ \\
\hline $\mathrm{Cu}^{\mathrm{vi}}-\mathrm{O} 3-\mathrm{K}^{\mathrm{vi}}$ & $94.34(7)$ \\
\hline $\mathrm{Na}^{\mathrm{iii}}-\mathrm{O} 3-\mathrm{K}^{\mathrm{vi}}$ & $163.09(7)$ \\
\hline $\mathrm{K}^{\mathrm{v}}-\mathrm{O} 3-\mathrm{K}^{\mathrm{vi}}$ & $85.14(5)$ \\
\hline $\mathrm{P} 2-\mathrm{O} 4-\mathrm{P} 1$ & $119.01(11)$ \\
\hline $\mathrm{P} 2-\mathrm{O} 5-\mathrm{Cu}^{\mathrm{ix}}$ & $128.83(11)$ \\
\hline $\mathrm{P} 2-\mathrm{O} 5-\mathrm{Na}$ & $106.28(10)$ \\
\hline $\mathrm{Cu}^{\mathrm{ix}}-\mathrm{O} 5-\mathrm{Na}$ & $86.95(7)$ \\
\hline $\mathrm{P} 2-\mathrm{O} 5-\mathrm{K}^{\mathrm{vii}}$ & $139.70(11)$ \\
\hline $\mathrm{Cu}^{\mathrm{ix}}-\mathrm{O} 5-\mathrm{K}^{\mathrm{vii}}$ & $87.32(5)$ \\
\hline $\mathrm{Na}-\mathrm{O} 5-\mathrm{K}^{\mathrm{vii}}$ & $90.88(6)$ \\
\hline $\mathrm{P} 2-\mathrm{O} 5-\mathrm{Na}^{\mathrm{vi}}$ & $86.16(8)$ \\
\hline $\mathrm{Cu}^{\mathrm{ix}}-\mathrm{O} 5-\mathrm{Na}^{\mathrm{vi}}$ & $80.94(6)$ \\
\hline $\mathrm{Na}-\mathrm{O} 5-\mathrm{Na}^{\mathrm{vi}}$ & $166.46(9)$ \\
\hline
\end{tabular}

$92.89(8)$

$126.37(7)$

$111.80(8)$

$116.12(7)$

$112.48(7)$

$150.23(8)$

$79.37(6)$

$72.83(6)$

$97.87(7)$

$85.37(7)$

$67.10(6)$

$166.46(9)$

$56.39(5)$

$114.42(7)$

$91.46(7)$

$56.27(5)$

$70.10(6)$

$156.06(5)$

$59.34(5)$

123.32 (6)

93.49 (6)

94.77 (5)

138.41 (6)

29.06 (4)

114.67 (5)

28.80 (4)

$150.56(5)$

96.04 (6)

28.24 (4)
26.06 (4)

108.15 (4)

92.83 (4)

102.49 (2)

$139.21(4)$

41.57 (4)

$93.78(5)$

34.34 (3)

34.63 (3)

$101.36(5)$

$91.51(4)$

141.53 (4)

115.54 (2)

16.45 (2)

$53.68(14)$

08.83 (11)

93.05 (7)

$92.08(9)$

86.20 (7)

126.29 (7)

$125.16(11)$

$113.55(8)$

6.03 (8)

28.21 (9)

92.88 (5)

$26.56(11)$

$106.61(10)$

85.17 (8)

$98.72(8)$

$134.68(8)$

83.27 (6)

87.28 (9)

94.34 (7)

(63.09 (7)

119.01 (11)

$28.83(11)$

$106.28(10)$

86.95 (7)

$139.70(11)$

$87.32(5)$

90.88 (6)

$86.16(8)$

166.46 (9) 


\begin{tabular}{|c|c|c|c|}
\hline $\mathrm{O} 5-\mathrm{Na}-\mathrm{P} 2^{\text {viii }}$ & $98.55(5)$ & 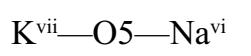 & $82.58(6)$ \\
\hline $\mathrm{O} 7^{\mathrm{i}}-\mathrm{Na}-\mathrm{P} 2^{\text {viii }}$ & $136.17(6)$ & $\mathrm{P} 2-\mathrm{O} 6-\mathrm{Cu}$ & $126.10(11)$ \\
\hline $\mathrm{O}^{\text {viii }}-\mathrm{Na}-\mathrm{P} 2^{\text {viii }}$ & $61.77(4)$ & $\mathrm{P} 2-\mathrm{O} 6-\mathrm{Na}^{\mathrm{iii}}$ & $103.71(9)$ \\
\hline $\mathrm{O} 5^{\mathrm{i}}-\mathrm{Na}-\mathrm{P} 2^{\text {viii }}$ & $94.99(5)$ & $\mathrm{Cu}-\mathrm{O} 6-\mathrm{Na}^{\mathrm{iii}}$ & $99.80(7)$ \\
\hline $\mathrm{O} 7^{\text {viii }}-\mathrm{Na}-\mathrm{P} 2^{\text {viii }}$ & $29.06(3)$ & $\mathrm{P} 2-\mathrm{O} 6-\mathrm{K}^{\mathrm{iv}}$ & $134.97(10)$ \\
\hline $\mathrm{P} 2^{\mathrm{i}}-\mathrm{Na}-\mathrm{P} 2^{\mathrm{viii}}$ & $121.50(4)$ & $\mathrm{Cu}-\mathrm{O} 6-\mathrm{K}^{\mathrm{iv}}$ & $92.63(6)$ \\
\hline $\mathrm{O} 1^{\mathrm{vii}-\mathrm{Na}-\mathrm{P} 2}$ & $99.77(6)$ & $\mathrm{Na}^{\mathrm{iii}}-\mathrm{O} 6-\mathrm{K}^{\mathrm{iv}}$ & $89.12(6)$ \\
\hline $\mathrm{O}^{\text {viii }}-\mathrm{Na}-\mathrm{P} 2$ & $151.35(6)$ & $\mathrm{P} 2-\mathrm{O} 7-\mathrm{Cu}^{\mathrm{vi}}$ & $124.98(11)$ \\
\hline $\mathrm{O} 5-\mathrm{Na}-\mathrm{P} 2$ & $27.01(4)$ & $\mathrm{P} 2-\mathrm{O} 7-\mathrm{Na}^{\mathrm{vi}}$ & $99.93(9)$ \\
\hline $\mathrm{O} 7^{\mathrm{i}}-\mathrm{Na}-\mathrm{P} 2$ & $85.78(5)$ & $\mathrm{Cu}^{\mathrm{vi}}-\mathrm{O} 7-\mathrm{Na}^{\mathrm{vi}}$ & $104.36(7)$ \\
\hline $\mathrm{O}^{\text {viii }-\mathrm{Na}-\mathrm{P} 2}$ & $79.46(5)$ & $\mathrm{P} 2-\mathrm{O} 7-\mathrm{K}^{\mathrm{xii}}$ & $137.96(10)$ \\
\hline $\mathrm{O} 5^{\mathrm{i}}-\mathrm{Na}-\mathrm{P} 2$ & $140.25(5)$ & $\mathrm{Cu}^{\mathrm{vi}}-\mathrm{O} 7-\mathrm{K}^{\mathrm{xii}}$ & $90.92(6)$ \\
\hline $\mathrm{O} 7^{\text {viii_- }} \mathrm{Na}-\mathrm{P} 2$ & $96.10(4)$ & $\mathrm{Na}^{\mathrm{vi}}-\mathrm{O} 7-\mathrm{K}^{\mathrm{xii}}$ & $89.80(6)$ \\
\hline $\mathrm{P} 2 \mathrm{i}-\mathrm{Na}-\mathrm{P} 2$ & $111.56(3)$ & $\mathrm{P} 2-\mathrm{O} 7-\mathrm{Na}^{\mathrm{iii}}$ & $84.73(7)$ \\
\hline $\mathrm{P} 2^{\text {viii }-\mathrm{Na}-\mathrm{P} 2}$ & $123.17(4)$ & $\mathrm{Cu}^{\mathrm{vi}}-\mathrm{O} 7-\mathrm{Na}^{\mathrm{iii}}$ & $81.55(6)$ \\
\hline $\mathrm{O}^{\mathrm{vii}}-\mathrm{Na}-\mathrm{Cu}^{\mathrm{ix}}$ & $115.94(6)$ & $\mathrm{Na}^{\mathrm{vi}}-\mathrm{O} 7-\mathrm{Na}^{\mathrm{iii}}$ & $167.87(8)$ \\
\hline $\mathrm{O}^{\mathrm{viii}}-\mathrm{Na}-\mathrm{Cu}^{\mathrm{ix}}$ & $86.18(5)$ & $\mathrm{K}^{\mathrm{xii}}-\mathrm{O} 7-\mathrm{Na}^{\mathrm{iii}}$ & $79.43(5)$ \\
\hline
\end{tabular}

Symmetry codes: (i) $x+1, y, z$; (ii) $x+1 / 2,-y+3 / 2, z+1 / 2$; (iii) $x-1 / 2,-y+3 / 2, z+1 / 2$; (iv) $-x+3 / 2, y+1 / 2,-z+1 / 2$; (v) $-x+1,-y+1,-z+1$; (vi) $x-1, y, z$; (vii) $-x+1,-y+1,-z$; (viii) $x+1 / 2,-y+3 / 2, z-1 / 2$; (ix) $x-1 / 2,-y+3 / 2, z-1 / 2$; (x) $-x+3 / 2, y-1 / 2,-z+1 / 2$; (xi) $-x+1 / 2, y-1 / 2,-z+1 / 2$; (xii) $-x+1 / 2, y+1 / 2,-z+1 / 2$. 